\title{
Two Autopsy Cases of Duplication of the Ureter
}

\author{
By

\begin{abstract}
Masatoshi Fuyuta, Yukihiko Miyayama*,
\end{abstract} \\ Mizuho Mochinaga ${ }^{* *}$ and Toyoaki Fujimoto \\ Department of Anatomy, Kumamoto University Medical School \\ Kumamoto 860 , Japan \\ -Received for Publication, October 11, 1975-

\section{Introduction}

Development of the urinary system in man and mammals follows such a complex process and is so intimately related with the development of the genital system that various anomalies are apt to appear. Among these, duplication of the ureter is found rather frequently (Nation 19441, Warkany 1971). Duplication of the ureter is classified into a complete type with doubling of both the renal pelvis and the ureter in the entire course to the bladder and an incomplete type with doubling of the renal pelvis and part of the ureter alone. The incomplete type is found more frequently than the complete type and the unilateral ty pe more frequently than the bilateral ty pe (Takahashi et al. $1928^{8}, 1932^{4}$, Horigome $1960^{\circ}$ ). These anomalies are often detected in clinical urography for the renal pelvis and ureter, but detection during body dissection is also common (Tsukahara 1954', Yamamoto 1955?, Kazama et al. $1958^{8.9}$.

We have experienced 2 cases of duplication of the renal pelvis and ureter upon dissection in the Kumamoto University Medical School, as reported below.

\section{Findings}

Case 1. The anormaly was found on the left side of a femal cadaver, with two ureters (duplicated ureters) emerging from the left renal hilus behind the renal artery and vein in upward and downward alignment (named superior and inferior ureter, respectively) (Fig. I-1). These

\footnotetext{
Present address :

* Training School for Medical Technologists, affiliated with Kumamoto University Medical School, Kumamoto, Japan.

** Department of Surgery, Kumamoto University Medical School, Kumamoto, Japan.
} 
ureters subsequently descended in a parallel course the inferior ureter occupying the lateral and the superior ureter, the medial aspect. Near the bladder, these two crossed each other, the superior ureter assuming the inferior and the inferior ureter, the superior position, each opening into the bladder (described subsequently).

The left kidney measured $11.1 \mathrm{~cm}$ in length, $6.1 \mathrm{~cm}$ in width, $5.4 \mathrm{~cm}$ in thickness, and weighed $150 \mathrm{~g}$, giving an impression of being somewhat enlarged longitudinally. Observation of the inside of the kidney which was sectioned frontally into approximately equal parts revealed duplication of the renal pelvis occupying the upper and lower parts of the kidney (each tentatively called superior and inferior renal pelvis) (Fig. I-2). The superior ureter emerged from the superior renal pelvis and the inferior ureter from the inferior pelvis, respectively.

In the lower part of the ureter near the bladder, the duplicated ureters were close to each other, and their separation could not be readily confirmed from the outside, therefore cross-sectional specimens were examined. Fig. I-3 shows the transverse section immediately before the entrance of the ureter into the bladder wall, indicating a complete separation of the two ureters. These reduplicated ureters passed through the bladder wall independently of each other. As was already described, the superior and inferior ureters crossed near the bladder, so that the opening of the superior ureter into the bladder wall was located inferiorly and that of the inferior ureter was seen superiorly. The opening of the superior ureter was found at approximately the same level as that of the single ureter on the right side (Fig. I-4).

From these findings this anomaly falls under the complete type of duplication of the renal pelvis and ureter on the left side.

Case 2. This anomaly was found in a female cadaver at the age of 77. In both the left and right kidneys, two dilated ureters, superior and inferior, arose from the renal hilus (Fig. II-1). On both the left and right sides, these ureters joined into one shortly after leaving the kidney, exhibiting the type of so-called incomplete double ureter. As described subsequently, the renal pelvis was duplicated in both the left and right kidneys (superior and inferior renal pelvis) associated with hydronephrosis. The findings are described for each side, subsequently.

i) The left kidney measured $9.6 \mathrm{~cm}$ in length, $5.5 \mathrm{~cm}$ in width, $3.3 \mathrm{~cm}$ in thickness, and weighed $51 \mathrm{~g}$, less than the normal kidney. From the renal hilus, markedly dilated double renal pelves described above protruded outside of the kidney, compressing the renal artery and vein anteriorly and superiorly. Each ureter extending from these was also dilated, and the two ureters joined at about $9 \mathrm{~cm}$ inferior to the renal hilus, forming one ureter (Figs. II-1,2). In the frontal section passing 
through the renal hilus, complete duplication of the renal pelvis was well demonstrated. Tho septum between the superior and inferior pelves was extremely thin, and both renal pelves and calices were markedly dilated; while renal parenchyma showed marked atrophy and remained only slightly in the periphery (Fig. II-2). Observation of the tissue specimen taken from the same site showed marked devastation of tubular structures and hyaline degeneration of glomeruli (Fig. II-3), indicating the presence of hydronephrosis.

ii) The right kidney measured $9.2 \mathrm{~cm}$ in length, $5.5 \mathrm{~cm}$ in width, $4.1 \mathrm{~cm}$ in thickness and weighed $106 \mathrm{~g}$, with the outside appearance scarcely different from the normal one. Two ureters emerging from the renal hilus immediately joined $(2 \mathrm{~cm}$ below) to form one ureter. In the frontal cut surface passing through the renal hilus (Fig. II-4), duplication of the renal pelvis was noted as in the left side. The inferior renal pelvis and calices were slightly dilated, and the histological examination also revealed mild hydronephrosis.

As to the opening of the ureter into the bladder, stenosis was noted about $5 \mathrm{~cm}$ above the entrance to the bladder on the left ureter, with dilation of the portion above this level (Fig. II-5). The bladder wall was markedly thickened and sclerotic, and the left ureter passed through the thickened wall. Observation of the tissue of this site, histologically, found no normal mucosa with occupation of the lumen by a tissue mass probably representing proliferating transitional epithelial carcinoma, with resulting obstruction (Fig. II-6).

The sclerosed, thickened bladder wall described above was explained by infiltration of this carcinoma. On the other hand, no remarkable changes, as seen on the left side, were noted in the right ureter and bladder wall.

Based on these findings the anomaly in case 2 consists of bilateral complete double renal pelvis, and incomplete double ureter, with association of hydronephrosis. Hydronephrosis in the left kidney may be due to stenosis of the distal portion of the ureter on the same side by proliferated carcinoma tissue.

\section{Discussion}

Development of the human ureter starts in the 5th week of fetal life slightly caudal to the opening of the Wolffian duct to the cloaca as a urcteric bud expanding from its dorsal side (Fig. III-1A). The bud thereafter gradually extends dorsally and cranially, reaching the metanephric blastema. On the other hand, at the original portion of the ureteric bud, with further development, a more complicated differentiation takes place. Through formation of the urorectal septum, the ventral side of the cloaca is separated as the urogenital sinus; and the opening 
of the ureteric bud gradually moves caudally over the Wolffian duct. The lower end of the future ureter and Woffian duct drain into a common chamber appearing as a bulge of the posterior wall of the urogenital sinus (Fig. III-1B). In the 7th week, the ureter and Wolffian duct are separated, each obtaining independent orifices. At first the openings of these two are close together. Subsequently and along with the differentiation of the urogenital sinus, the ureteral orifice gradually moves cranially and laterally, while the Wolffian duct shifts caudally (Fig. III1C). In the 8th week, the former opens at the subsequent bladder and the latter at the subsequent urethra (Fig. III-1D). In view of such a course of development, two ureteric buds from the lower end and posterior wall of the Wolffian duct appear unilaterally (Fig.,III-2A), giving rise to the complete double ureter upon reaching the motanephric blastema. If each of the ureteric buds described above accomplish their differentiation, the renal pelvis would also become double. Such an excess ureteric bud probably participates in the formation of the double ureter, although the detail of its mechanism is still a question of understanding.

With the ureteric buds appearing in double, first the inferior ureter starts to move cranially and laterally at the origin after separation from the Wolffian duct (Figs. III-2B, C). Since the superior ureter separates later than this, the ureter which was located lower at first is at the superior and lateral portion of the bottom of the bladder, while the ureter which was located higher at first opens in the inferior and medial aspect in the adult form. Two ureters originating from the superior and inferior renal pelves are said to cross each other (Weigert-Meyer's Law in Takahashi et al., 1932, Fig. III-2C). In our case 1, a complete double ureter was found on the left side, with the course prabably in agreement with the so-called W-M law described above. When two ureteric buds occur in extreme proximity, however, some are said to go against this law (Lund 1949 ${ }^{10}$ ).

As to the opening of the ureter into the bladder, as described above, the ureter from the superior pelvis keeps the connection with the Wolffian duct longer than the other ureter in the course of development, so that insufficient separation from the Wolffian duct occurs more frequently. As the result of such insufficient separation, the ureter opens into the seminal duct, ejaculatory duct or seminal vesicle in male (Fig. III-2D). When the separation described above occurs late, and the shift to the cranial and lateral direction is incomplete, the ureter ends near the opening of the Wolffian duct, and the opening to the bladder occurs near the urethra or close to it. Consequently, the occurrence of an opening abnormality of a complete double ureter, in case it occurs, should be frequently seen in the ureter originating from the superior renal pelvis. In our case 1 , it was the ureter originating from the 
superior pelvis that opened at a level approximately the same as the normal single ureter on the right side (Fig. I-4 should be referred to again). On the other hand, the ureter from the inferior pelvis opened into the bladder cranial and lateral to the normal site.

Incomplete double ureter may occur either as the incomplete double ureter with double pelvis through addition of an extra embryonal bud on one normal ureteric bud occurring unilaterally (Ohtaguro 1969 ${ }^{11}$ ) or as the result of early separation of the end of the ureteric bud which originally has the potency for separation and subsequently producing a double ureter at various levels between the Wolffian duct and the metanephric blastema (Nation ${ }^{1}$. In our case 2, a bilateral incomplete double ureter was found with the site of branching (site of doubling) close to the renal hilus at a considerably high level. This might indicate that before the ureteric bud reached the metanephric tissue, the tip was separated due to formation of two renal pelves.

\section{Summary}

Two cases of double ureter experienced upon body dissection in the Kumamoto University Medical School were reported :

1) Case 1: In a female cadaver, the renal pelvis was duplicated in the left kidney with associated complete ureteral duplication which continued into the bladder base with independent ureteral openings. Since the two ureters cross in the lower part, the original positional relationship between these two ureters was reversed at the opening site.

2) Case 2: In a 77 year-old female cadaver, bilateral double renal pelves were noted. The ureters which continued from the pelves also doubled only at a higher level (incomplete double ureter). In this case, obstruction of the left ureter was noted close to the opening at the bladder, probably due to infiltration of the carcinoma tissue. Marked hydronephrosis was noted in the kidney on the same side.

3) An embryological discussion was presented on the normal development of the ureter and the appearance of a double ureter.

\section{References}

1) Nation, E.F.: Duplication of the kidney and ureter: A statistical study of 230 new cases. J. Urol., $51:$ 456-465, 1944.

2) Warkany, J. : “Congenital Malformations," 1067-1073, Malformations of urogenital system. The ureter. Year Book Medical Publishers, Chicago, 1971.

3) Takahashi, A. and Ichikawa, T.: Vier Fälle von Nierenbecken- und Ureterverdoppelung (in Japanese). Jap. J. Dermatol. and Urol., 28 : 902-917, 1928.

4) ditto: Angeborene Nieren- und Uretermissbildungen. Über die Nierenbecken und Ureterverdoppelung. (2) (in Japanese). Jap. J. Dermatol. and Urol., 32: 
16-39, 1932.

5) Horigome, S.: Studies on malformation of urogenital tract. Report 2. Statistical observation (in Japanese). Jap. J. Urol., 51 : 567-573, 1960.

6) Tsukahara, K.: On a case of double ureter (in Japanese). Reports from the Department of Anatomy, Tokyo Jikeikai School of Medicine, 11, 1954.

7) Yamamoto, T.: One case of the complete duplication of ureter and double pelvis on the both sides (in Japanese). Igaku Kenkyu (Acta Medica) Fukuoka, Japan. 25: 1039-1041, 1955.

8) Kazama, Y. and Ichinohe, S.: Two cases of duplicated ureter (in Japanese). Reports from the Department of Anatomy, Iwate Medical School, 5 : 11-14, 1958.

9) Kazama, Y., Endo, K. and Hwang, Mu-Su.: On a case of complete duplication of the ureter (in Japanese). Reports from the Department of Anatomy, Iwate Medical School, 5 : 99-102, 1958.

10) Lund, A. J.: Uncrossed double ureter with rare intraversical orifice relationship : Case report with review of literature. J. Urol., 62: 22-29, 1949.

11) Ohtaguro, K. : Congenital anomalies of ureter (in Japanese). Jap. J. Clin. Urol., 23 : 353-362, 1969.

12) Tuchmann-Duplessis, H. and Haegel, P.: Organogenesis: Urinary system. In "Illustrated human embryology, Vol. 2." Translated by Hurley, L.S. SpringerVerlag, New York, p. 50-71, 1972.

\section{Explanation of Figures}

\section{Plate I}

\section{Case 1.}

Fig. I-1: Complete double ureter on the left side. The parallelling superior ureter (Us) descends on the medial side of the inferior ureter (Ui). Both cross near the bladder (arrow). $0.25 \times$.

Fig. I-2 : Dorsal view of the ventral half of the left kidney cut in the frontal plane. The renal pelvis is also completely duplicated ( $\mathrm{Ps}$ and $\mathrm{Pi}$ ). $0.5 \times$.

Fig. I-3: Transverse section of parallel double ureter at the portion near the entrance into the bladder, H. and E. stain. $16 \times$.

Fig. I-4: Inside view of the trigonum vesicae; three ureteral orifices open at the base of the bladder (arrows). On the left side, the ureter from the superior pelvis (So) opens more caudally and more centrally than the ureter from the inferior pelvis (Io). $2.5 \times$. 
Plate I

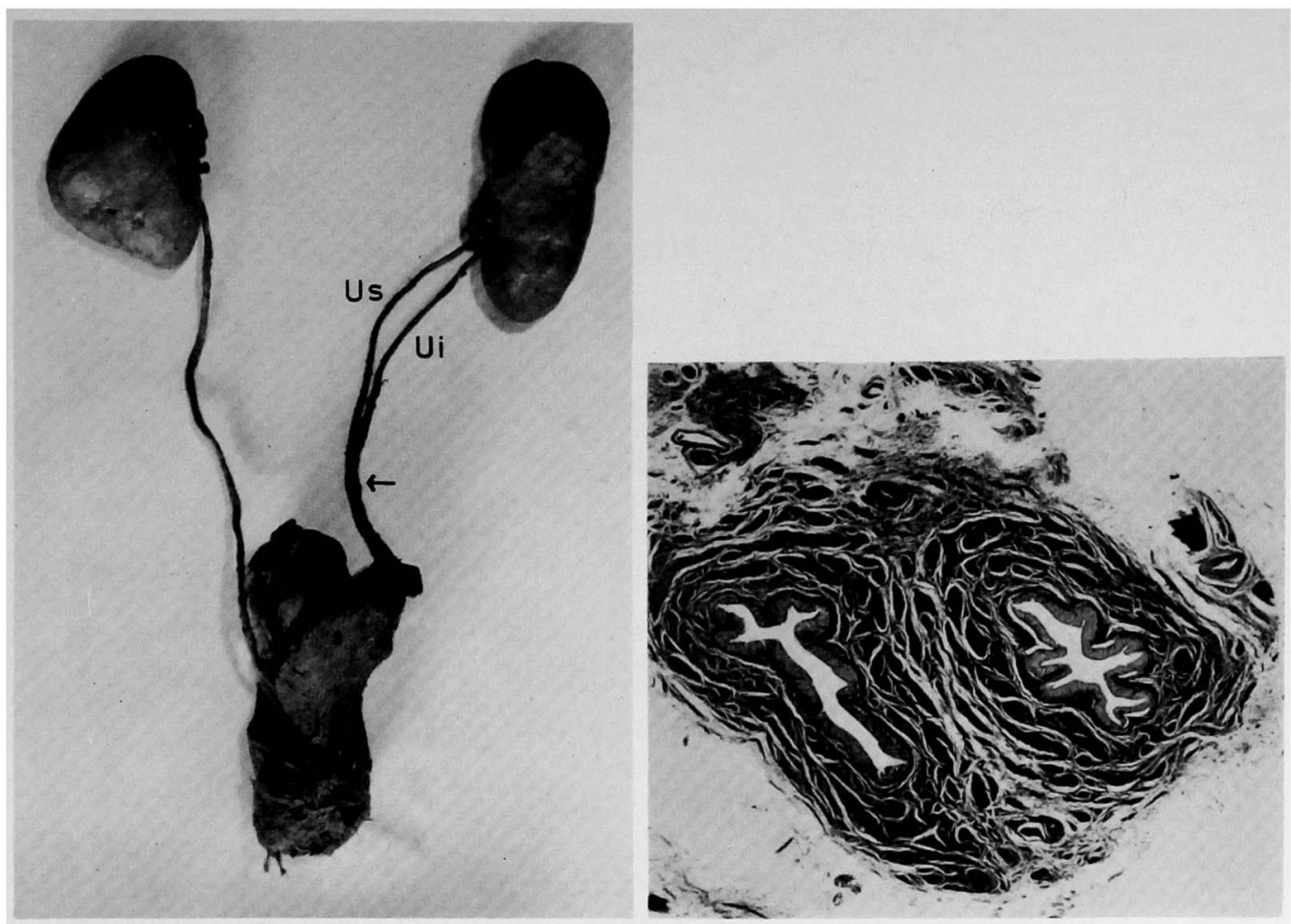

1

2
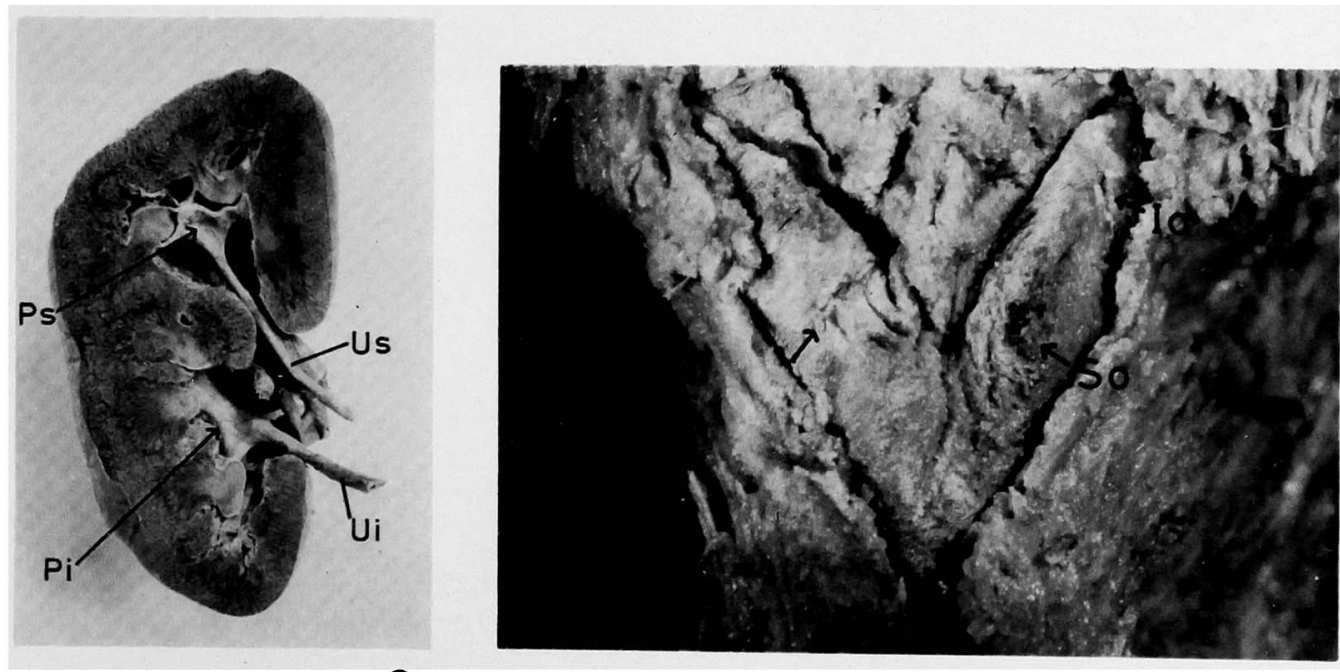

3

Fig. I

M.Fuyuta et al. 


\section{Plate II}

Case 2.

Fig. II-1: Bilateral incomplete double ureter (arrows), ventral view. A, V: A. et $\mathrm{V}$. renalis, $\mathrm{L}$ : left kidney, $\mathrm{R}$ : right kidney.

Fig. II-2: Shows the inside of the left kidney cut frontally through the hilus into approximately equal parts. The renal pelvis is duplicated and dilated along with the calyces (Hydronephrosis). $0.33 \times$.

$\mathrm{Ps}$ : superior pelvis, $\mathrm{Pi}$ : inferior pelvis, Us : superior ureter, Ui : inferior ureter.

Fig. II-3: Histological section of the left renal cortex. Devastation of urinary tubules, and hyaline degeneration of glomeruli (arrows). H. and E. stain. $100 \times$.

Fig. II-4: Represents the inside of the right kidney with duplicated pelvis, which is cut frontally through the hilus. $0.33 \times$.

Ps : superior pelvis, $\mathrm{Pi}$ : inferior pelvis, Us : superior ureter, $\mathrm{Ui}$ : inferior ureter.

Fig. II-5: Upper half of the urinary bladder; the left lateral (arrow-1) and posterior (arrow-p) walls of the bladder are thickened. The dilated left ureter is abruptly narrowed at the end portion indicated by the large arrow.

Fig. II-6: Histological picture of the transverse section of the site of stenosis of Fig. II-5 (large arrow), indicating ureteral obstruction by proliferation of the carcinoma tissue $(\mathrm{Ct})$. H. and E. stain. $6 \times$. 
Plate II

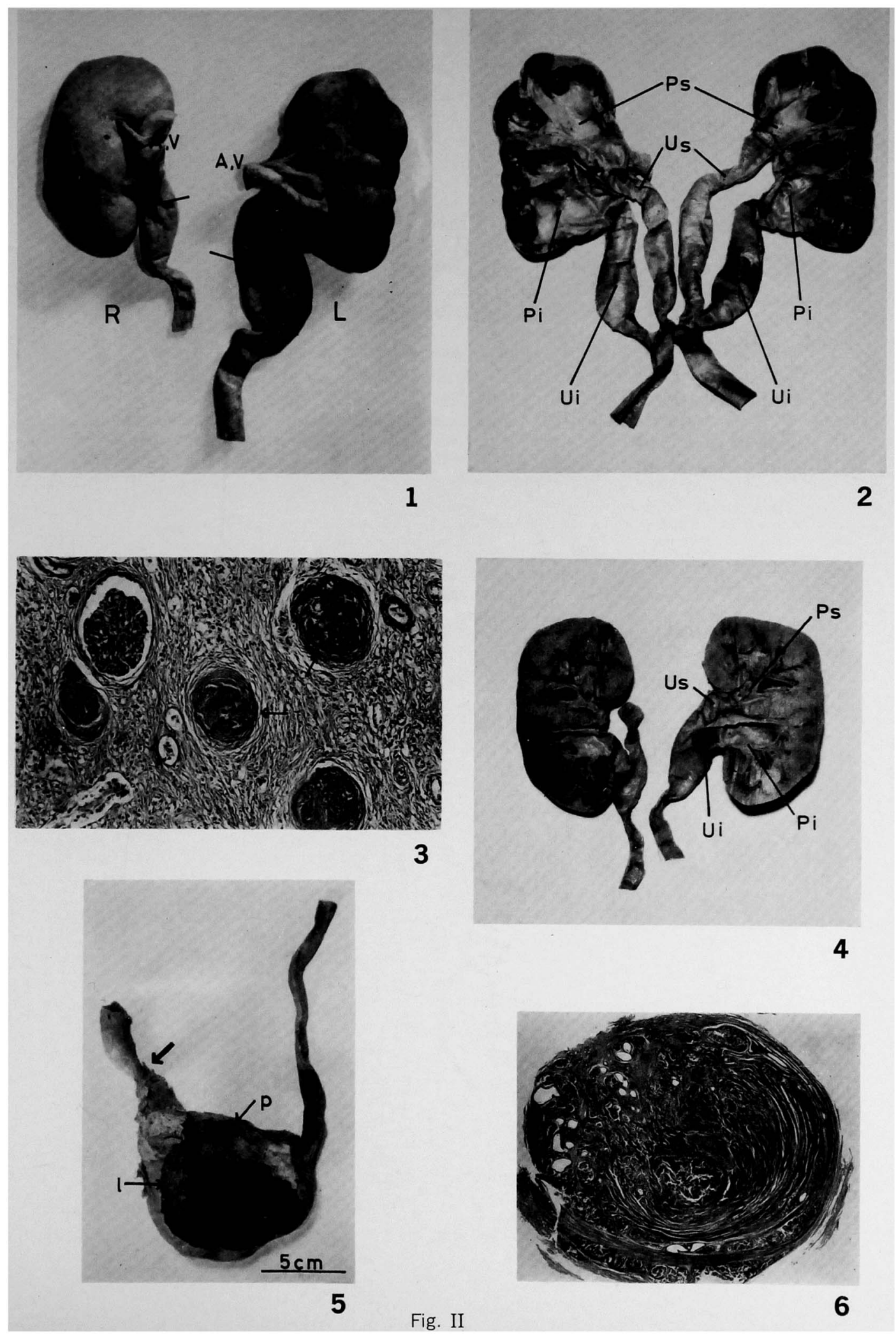




\section{Plate III}

Fig. III-1 : Normal development of the ureter and its surroundings.

(redrawn from Tuchmann-Duplessis, H. and et al, Illustrated Human Embryology, Vol. 2, Fig. 1 on page 66, Springer-Verlag, New York, 1972 ${ }^{12}$ ).

Fig. III-2: Embryological explanation of the occurrence of double ureter. (redrawn from Nation, E.F., J. Urol. 51, Fig. 2 on page 458, 1944.) Abbreviations in Fig. III : $\mathrm{Bl}$ : bladder, $\mathrm{C}$ : cloaca, Ed : ejaculatory duct, $\mathrm{G}$ : gut, $\mathrm{K}$ : kidney, U: ureter, Ub: ureteric bud, Vua : vesico-urethral anlage, W : Wolffian duct. 
283

Plate III
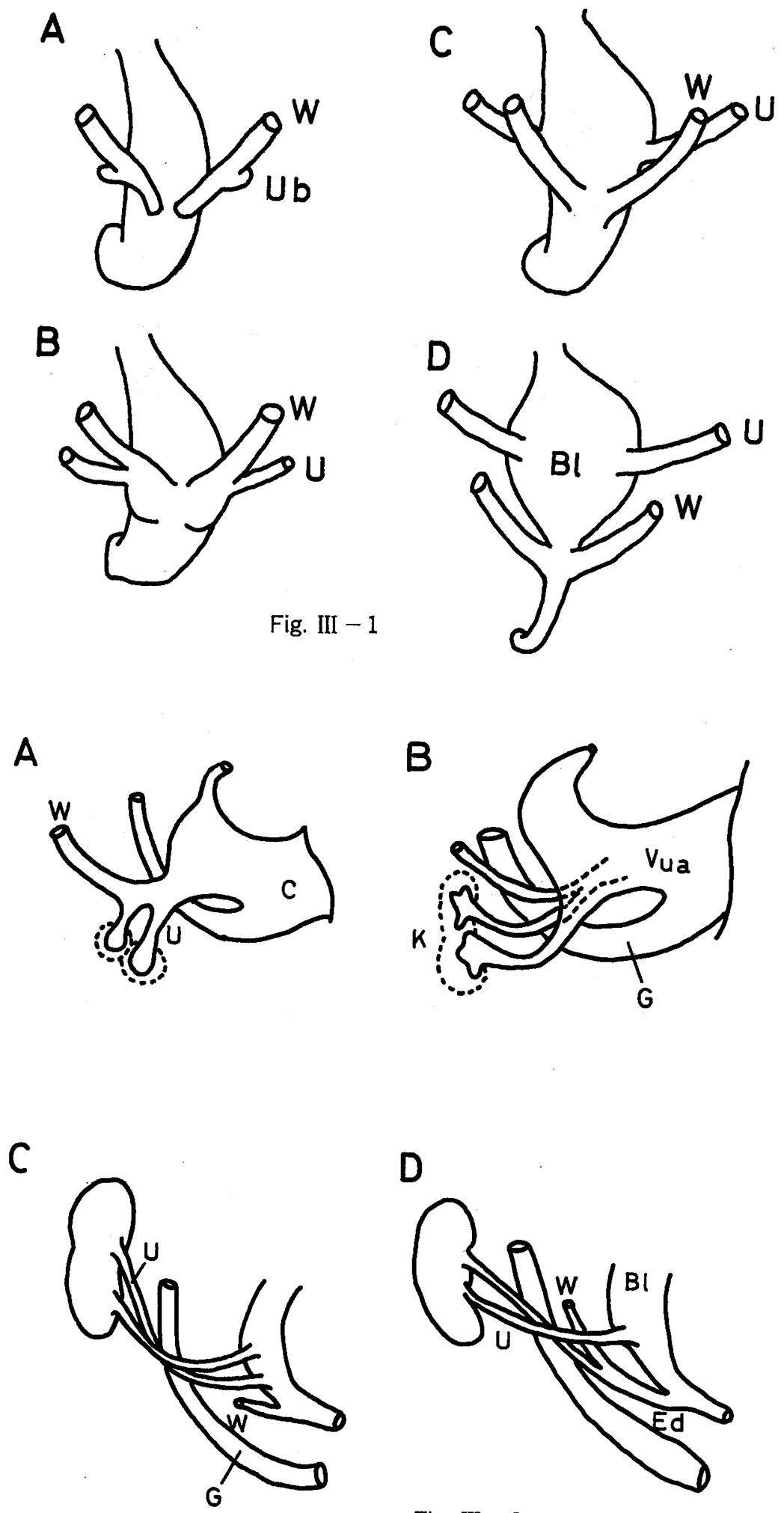

Fig. III -2

M.Fuyuta et al. 